\title{
La dinámica de la pobreza de trabajadores ${ }^{1}$
}

\author{
Rodolfo Gutiérrez \\ Universidad de Oviedo \\ rgutier@uniovi.es
}

\section{Resumen}

En este artículo se ofrece una primera aproximación al análisis dinámico de la pobreza de los ocupados en España a partir de microdatos de la European Union-Survey on Income and Living Conditions y la Encuesta de Condiciones de Vida en España, con un posterior estudio empírico centrado, en primer lugar, en la descripción de la persistencia laboral $y$ los flujos de entrada y salida de la misma en un grupo de países europeos; $y$, en segundo lugar, en el análisis de los determinantes de dicha movilidad en el caso español, con especial atención a la influencia de los cambios en la composición de los hogares y en la participación laboral de sus miembros.

Palabras clave: dinámicas de pobreza, pobreza laboral, desigualdad.

\begin{abstract}
This article provides an initial approach to the dynamic analysis of the poverty of workers in Spain based on microdata from the European Union Survey on Income and Living Conditions and the Encuesta de Condiciones de Vida en España, and a subsequent empirical study that focuses, first, on describing employment persistence and entry and exit flows in a group of European countries and, second, on analyzing the determinants of this mobility in the case of Spain. Particular attention is paid to the influence of changes in the composition of housebolds and in the time spent in employment of their members.
\end{abstract}

Key words: poverty dynamic, working poverty, inequality.

\footnotetext{
1 Este trabajo es resultado de las actividades desarrolladas dentro del proyecto de investigación que ha sido financiado por un proyecto del Plan Nacional del I+D+I «Trabajadores pobres» (SEJ2005-8464) y de la Red de Excelencia RECWOWE del VI Programa Marco (UE-06-828339). En este proyecto colaboran también Isabel García, Marta Ibáñez y Aroa Tejero.
} 


\section{Introducción}

En el estudio de los trabajadores pobres confluyen dos tipos de situaciones, el empleo y la pobreza, que habitualmente han sido analizadas de manera separada. Desde el punto de vista conceptual, la pobreza laboral es una categoría híbrida que se define operativamente por la presencia simultánea en los individuos de cierto nivel de participación en el trabajo y de una situación de pobreza de los hogares.

$\mathrm{El}$ asunto de los trabajadores pobres ha ido adquiriendo una creciente relevancia en los últimos años, motivada por la confluencia en este tema de dos tipos de intereses: de una parte, la propia preocupación por un posible aumento de la desigualdad de rentas en la época más reciente, preocupación que se haría mayor de confirmarse que el riesgo de pobreza crece entre personas ocupadas; de otra, la atención a las consecuencias del cambio de orientación de las políticas de inclusión social hacia un enfoque centrado en el empleo, frente al más tradicional, que se centraba en las transferencias sociales. A ello se añade la disponibilidad de nuevas fuentes estadísticas que proporcionan algunas oportunidades de mejora del conocimiento empírico de esas situaciones.

Los estudios sobre la dinámica de la pobreza suelen mostrar que existe una gran movilidad en la zona baja de la distribución de la renta, de manera que el porcentaje de personas que experimentan algún periodo de pobreza a lo largo de su vida, o en alguno de los años de un determinado periodo, es muy superior al de los afectados por la pobreza en un año específico o en el promedio de varios años. Sin embargo, son muy escasos los estudios sobre dinámicas de pobreza que presten atención específica a la pobreza de trabajadores.

En esta comunicación se ofrece una primera aproximación al análisis dinámico de la pobreza de ocupados en España, con alguna referencia comparada, a partir de microdatos de la European Union-Survey on Income and Living Conditions (UE-SILC) y de la Encuesta de Condiciones de Vida en España (ECV). En primer lugar, se ofrece un panorama de la investigación reciente sobre la dinámica de la pobreza; luego se presentan y comentan las posibilidades y limitaciones que ofrece la fuente de datos UE-SILC para el análisis dinámico de estas situaciones. Posteriormente, se realiza un estudio empírico centrado en dos tipos de cuestiones: en primer lugar, la descripción de la persistencia en la pobreza laboral y los flujos de entrada y salida en la misma en un grupo de países europeos; en segundo lugar, el análisis de los determinantes de dicha movilidad en el caso español, con especial atención a la influencia de los cambios en la composición de los hogares y en la participación laboral de sus miembros. 


\section{Los estudios sobre dinámica de pobreza}

Los estudios de corte transversal suelen mostrar que los niveles de pobreza no se reducen en el periodo más reciente y que las diferencias entre países se mantienen en buena medida en sus niveles relativos (OCDE, 2009a). Entre mediados de las décadas de 1980 y 1990, la tasa de pobreza (no ponderada) de 24 países de la OCDE se incrementó en 0,6 puntos; en la siguiente década, los cambios fueron de similar magnitud y dirección. En el periodo que va de mediados de los años ochenta hasta mediados de la década de 2000, la tasa de pobreza aumentó en dos terceras partes en el conjunto de países de la OCDE, que finalizaron el periodo con una tasa cercana al $11 \%$.

También la pobreza laboral se muestra muy estable a lo largo del periodo para el que hay datos disponibles, que coincide con el ciclo expansivo vivido por las economías europeas desde mediados de los años noventa (tabla 1). En el conjunto de la UE-15, la tasa de riesgo de pobreza de ocupados se ha mantenido en los últimos diez años entre el 7 y el $8 \%$. En una buena parte de países se observó una ligera reducción de ese indicador en los años finales de la década de 1990, pero ha retornado a cifras algo más altas en los años recientes. Entre los 25 países de la UE, tan sólo en los países bálticos y en Portugal parece registrarse una tendencia a la reducción de este indicador, aunque la ruptura de las series, o los pocos años de las mismas, hacen que se deba tomar con precaución esos cambios en el corto plazo. En el caso de España, la estabilidad es muy destacable, ya que el indicador oscila entre los valores 10 y 11 para todo el periodo 1996-2007.

Tabla 1. Tasa de riesgo de pobreza de ocupados (después de transferencias sociales) 19962007 (Pobreza basada en un 60\% de la media)

\begin{tabular}{|l|c|c|c|c|c|c|c|c|c|c|c|c|}
\hline & 1996 & 1997 & 1998 & 1999 & 2000 & 2001 & 2002 & 2003 & 2004 & 2005 & 2006 & 2007 \\
\hline EU-25 & $:$ & $:$ & $:$ & $:$ & $:$ & 8 & 8 & 8 & 8 & $8^{(\mathrm{b})}$ & 8 & $8^{(\mathrm{p})}$ \\
\hline EU-15 & $8^{(\mathrm{s})}$ & $8^{(\mathrm{s})}$ & $7^{(\mathrm{s})}$ & $7^{(\mathrm{s})}$ & $7^{(\mathrm{s})}$ & 8 & 8 & 8 & 8 & $7^{(\mathrm{b})}$ & 7 & $8^{(\mathrm{p})}$ \\
\hline Bélgica & 6 & 5 & 4 & 5 & 5 & 4 & $:$ & $6^{(\mathrm{b})}$ & 4 & 4 & 4 & 4 \\
\hline Bulgaria & $:$ & $:$ & $:$ & $:$ & $:$ & $7^{(\mathrm{i})}$ & $6^{(\mathrm{i})}$ & $7^{(\mathrm{i})}$ & $7^{(\mathrm{i})}$ & $6^{(\mathrm{i})}$ & $6^{(\mathrm{i})}$ & $:$ \\
\hline $\begin{array}{l}\text { República } \\
\text { Checa }\end{array}$ & $:$ & $:$ & $:$ & $:$ & $:$ & $3^{(\mathrm{i})}$ & $:$ & $:$ & $:$ & $3^{(\mathrm{b})}$ & 3 & 3 \\
\hline Dinamarca & $:$ & $:$ & $:$ & $:$ & $:$ & $3^{(\mathrm{i})}$ & $:$ & $5^{(\mathrm{b})}$ & 5 & 5 & 4 & 4 \\
\hline Alemania & 5 & 5 & 4 & 5 & 4 & 4 & $:$ & $:$ & $:$ & $5^{(\mathrm{b})}$ & 5 & $7^{(\mathrm{p})}$ \\
\hline Estonia & $:$ & $:$ & $:$ & $:$ & $10^{(\mathrm{i})}$ & $10^{(\mathrm{i})}$ & $9^{(\mathrm{i})}$ & $10^{(\mathrm{i})}$ & $9^{(\mathrm{b})}$ & 7 & 8 & 8 \\
\hline Irlanda & 5 & 5 & 5 & 5 & 7 & 7 & $:$ & $7^{(\mathrm{b})}$ & 7 & 6 & 6 & 6 \\
\hline Grecia & 15 & 15 & 13 & 14 & 13 & 13 & $:$ & $14^{(\mathrm{b})}$ & 13 & 13 & 14 & 14 \\
\hline España & 10 & 11 & 10 & 9 & 8 & 10 & $10^{(\mathrm{bi})}$ & $10^{(\mathrm{i})}$ & $11^{(\mathrm{b})}$ & 10 & 10 & 11 \\
\hline Francia & 7 & 7 & 7 & 7 & 8 & $7^{(\mathrm{b})}$ & $7^{(\mathrm{i})}$ & $:$ & $5^{(\mathrm{b})}$ & 6 & 6 & 6 \\
\hline Italia & 11 & 11 & 9 & 9 & 10 & 10 & $:$ & $:$ & $9^{(\mathrm{b})}$ & 9 & 10 & 10 \\
\hline Chipre & $:$ & $6^{(\mathrm{i})}$ & $:$ & $:$ & $:$ & $:$ & $:$ & $6^{(\mathrm{i})}$ & $:$ & $7^{(\mathrm{b})}$ & 7 & 6 \\
\hline
\end{tabular}




\begin{tabular}{|l|c|c|c|c|c|c|c|c|c|c|c|c|}
\hline Letonia & $:$ & $:$ & $:$ & $:$ & $13^{(\mathrm{i})}$ & $:$ & $:$ & $:$ & $:$ & $9^{(\mathrm{b})}$ & 11 & 10 \\
\hline Lituania & $:$ & $:$ & $:$ & $:$ & $14^{(\mathrm{i})}$ & $14^{(\mathrm{i})}$ & $:$ & $:$ & $:$ & $10^{(\mathrm{b})}$ & 10 & 8 \\
\hline Luxemburgo & 6 & 7 & 7 & 9 & 8 & 8 & $:$ & 7 & 9 & 10 & 10 & 9 \\
\hline Hungría & $:$ & $:$ & $:$ & $:$ & $6^{(\mathrm{i})}$ & $5^{(\mathrm{i})}$ & $4^{(\mathrm{i})}$ & 6 & $:$ & $10^{(\mathrm{b})}$ & 7 & 6 \\
\hline Malta & $:$ & $:$ & $:$ & $:$ & 6 & $:$ & $:$ & $:$ & $:$ & 5 & 4 & 4 \\
\hline Países Bajos & 6 & 6 & 6 & 6 & $6^{(\mathrm{ip})}$ & $5^{(\mathrm{ip})}$ & $5^{(\mathrm{ip})}$ & $6^{(\mathrm{ip})}$ & $:$ & $6^{(\mathrm{b})}$ & 4 & 5 \\
\hline Austria & 7 & 7 & 7 & 7 & 6 & 6 & $:$ & $8^{(\mathrm{b})}$ & 7 & 7 & 6 & 6 \\
\hline Polonia & $:$ & $:$ & $:$ & $:$ & $11^{(\mathrm{i})}$ & $11^{(\mathrm{i})}$ & $:$ & $:$ & $:$ & $14^{(\mathrm{b})}$ & 13 & 12 \\
\hline Portugal & 15 & 14 & 14 & 14 & 14 & 12 & $:$ & $:$ & $13^{(\mathrm{b})}$ & 12 & $11^{(\mathrm{p})}$ & 10 \\
\hline Rumanía & $:$ & $:$ & $:$ & $:$ & $14^{(\mathrm{i})}$ & $14^{(\mathrm{i})}$ & $14^{(\mathrm{i})}$ & 14 & $:^{(\mathrm{i})}$ & $:^{(\mathrm{i})}$ & $:^{(\mathrm{i})}$ & $:$ \\
\hline Eslovenia & $:$ & $:$ & $:$ & $:$ & $5^{(\mathrm{i})}$ & $5^{(\mathrm{i})}$ & $4^{(\mathrm{i})}$ & 4 & $:$ & $5^{(\mathrm{b})}$ & 5 & 5 \\
\hline Eslovaquia & $:$ & $:$ & $:$ & $:$ & $:$ & $:$ & $:$ & $:$ & $:$ & $9^{(\mathrm{b})}$ & 6 & 5 \\
\hline Finlandia & 3 & 4 & 4 & 5 & 5 & $4^{(\mathrm{bi})}$ & $4^{(\mathrm{i})}$ & $4^{(\mathrm{i})}$ & 4 & 4 & 4 & 5 \\
\hline Suecia & $:$ & $:$ & $:$ & $:$ & $:$ & $5^{(\mathrm{i})}$ & $:$ & $:$ & $6^{(\mathrm{b})}$ & 5 & 7 & 7 \\
\hline Reino Unido & 7 & 5 & 6 & 7 & 6 & 7 & 7 & 7 & $:$ & $8^{(\mathrm{b})}$ & 8 & 8 \\
\hline Croacia & $:$ & $:$ & $:$ & $:$ & $:$ & $:$ & $:$ & $9^{(\mathrm{i})}$ & $:$ & $:$ & $:$ & $:$ \\
\hline Turquía & $:$ & $:$ & $:$ & $:$ & $:$ & $:$ & $23^{(\mathrm{i})}$ & 23 & $:$ & $:$ & $:$ & $:$ \\
\hline Islandia & $:$ & $:$ & $:$ & $:$ & $:$ & $:$ & $:$ & $:$ & $7^{(\mathrm{b})}$ & $8^{(\mathrm{p})}$ & $7^{(\mathrm{p})}$ & 7 \\
\hline Noruega & $:$ & $:$ & $:$ & $:$ & $:$ & $:$ & $:$ & $4^{(\mathrm{b})}$ & 4 & 5 & 6 & 6 \\
\hline
\end{tabular}

$:=$ No disponible; $\mathrm{b}=$ Cambio en la serie; $\mathrm{p}=$ Dato provisional; $\mathrm{s}=$ Estimación Eurostat.

Fuente: EUROSTAT

Uno de los principales resultados de los estudios sobre la dinámica de la pobreza es que suele haber una gran movilidad en la zona baja de la distribución, de manera que el porcentaje de personas que experimentan algún periodo de pobreza a lo largo de su vida, o en alguno de los años de un periodo concreto, es muy superior al de los afectados por la pobreza en un año determinado o al promedio en varios años; esto segundo es lo que proporcionan los análisis indicadores estáticos de pobreza. Sin embargo, los estudios sobre pobreza laboral apenas se han llevado a cabo con perspectiva dinámica. No obstante, se puede asumir que también este tipo de pobreza puede poseer un alto grado de movilidad. Es más que probable que las diferencias en la frecuencia y la composición de la pobreza laboral observados en diferentes estudios (Peña y Latta, 2004; Allegre, 2008; Fraser y Goerne, 2008; García, Gutiérrez e Ibáñez, 2008; Andress y Lohman, 2008) respondan también a patrones diferentes de persistencia y transitoriedad de la misma. Por otra parte, la investigación sobre determinantes de la pobreza laboral (Lohman y Marx, 2008; Lohman, 2008) suelen confirmar que las características del sistema de protección social y las instituciones del mercado de trabajo son los factores que en mayor medida explican las diferencias en extensión y composición de este tipo de pobreza. Siendo así, la cuestión más relevante es si estos factores ejercen similar influencia en la explicación de la dinámica de la pobreza laboral y, por tanto, si los países con similares niveles y patrones dinámicos de pobreza laboral se corresponden con las variantes típicas de regímenes de bienestar. 
El panorama europeo sobre el estudio de la dinámica de la pobreza debe basarse hasta ahora en los estudios sobre la pobreza total, no específicamente sobre la pobreza de ocupados, y en las fuentes estadísticas disponibles para los años noventa, que alcanzan hasta donde llega la principal fuente de datos utilizada, el Panel de Hogares de la UE (EHP), sin que se disponga todavía de estudios basados en la nueva fuente de la UE-SILC para años más recientes.

Un punto de referencia en la investigación sobre dinámica de la pobreza se sitúa en los años ochenta, en los Estados Unidos, al disponer desde esas fechas de una fuente de datos longitudinales de suficiente calidad como el Panel Study of Income Dynamics (PSID), basado en entrevistas a una significativa muestra de familias norteamericanas desde 1968. En Europa, estas investigaciones se retrasan hasta los años noventa, cuando, por primera vez, Duncan et alii (1993) intentan comparar la duración de la pobreza en un grupo de países (Alemania, Suecia, Países Bajos, Luxemburgo) y la región francesa de Lorena utilizando varias fuentes de información. Estas investigaciones se impulsan extraordinariamente desde que, en 1994 y hasta 2001, EUROSTAT proporcione el EHP, que se ha convertido en la herramienta fundamental para el análisis de la dinámica de la pobreza, y que constituye la fuente estadística y el marco de referencia temporal de lo que se podía saber sobre la dinámica de la pobreza con perspectiva comparada hasta hace muy poco tiempo.

Esta investigación ha proporcionado resultados que se pueden agrupar en tres tipos de análisis: los flujos de entrada y salida en las situaciones de pobreza; la duración o persistencia de estas situaciones, y el efecto de diferentes cambios sobre movilidad y duración, principalmente los cambios en la demografía de los hogares, en la participación en el mercado de trabajo y en el flujo de rentas de diverso origen que reciben los hogares.

\subsection{Flujos de movilidad}

La movilidad se estudia habitualmente a través de las tasas de entrada y salida en situación de pobreza; siendo las tasas de entrada el porcentaje de las personas que, no siendo pobres en un determinado año, son pobres en el año siguiente, y las tasas de salida el flujo inverso, es decir, la proporción de quienes siendo pobres en un año, no lo son en el año siguiente. En conjunto, estos indicadores suelen mostrar que la movilidad de la pobreza es muy alta y variable entre países. Esa gran movilidad se expresa principalmente a través de las tasas de salida, que en 17 países de la OCDE se situaban cerca del $40 \%$ al comienzo de primera década del siglo xxi (gráfico 2); las tasas de entrada mostraban valores más bajos, entre el $3 \%$ y el $7 \%$. 
Gráfico 2. Tasas de entrada y salida en/de situaciones de pobreza (h. 2000)

(Pobreza basada en un umbral del $50 \%$ de la media)

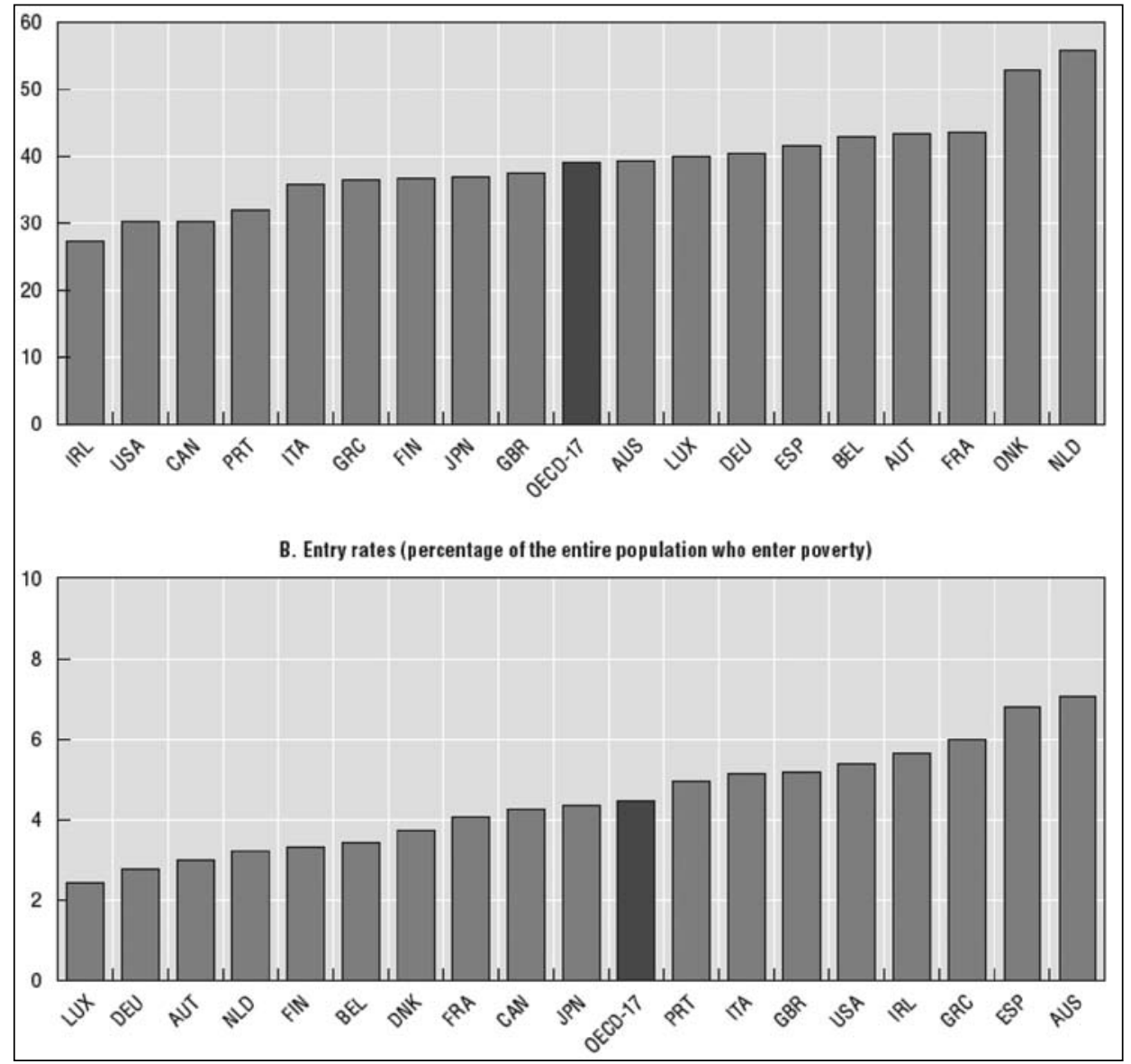

Fuente: Tomado de OCDE, 2009a, cap 6.

Otra forma habitual de analizar la movilidad de rentas consiste en observar las transiciones entre diferentes partes de la distribución. El mencionado estudio sobre 17 países de la OCDE (2009a) muestra que la movilidad es significativamente más baja para los hogares situados tanto en la parte más baja como en la más alta de la distribución de la renta de los hogares; casi el 70\% de las personas situadas en los dos quintiles más bajos permanecen en los mismos tras un periodo de tres años, y la mayoría de los que se mueven, lo hacen escalando uno o dos quintiles como máximo. De modo similar, fijándose en quienes son pobres en el año inicial, un promedio del 55\% aún están bajo el umbral de pobreza en el año final de los tres que se observan. También en promedio, para ese conjunto de la OCDE, sólo un $8 \%$ de quienes son pobres el primer año 
superan el umbral de pobreza en el año final; para más de la mitad de éstos, las rentas que consiguen no superan el $75 \%$ de la media al final de ese periodo de tres años.

\subsection{Persistencia en la pobreza}

Las medidas de la duración de las situaciones de pobreza permiten una mejor comprensión de los riesgos que afrontan las personas en situaciones de baja renta. El estudio de la duración de la pobreza se ha centrado fundamentalmente en la cuantificación y la caracterización de sus componentes persistente (o crónico) y temporal (o transitorio) en periodos de medio o largo plazo. Pero los conceptos de cronicidad y transitoriedad son susceptibles de diferentes enfoques metodológicos que responden a distintas estrategias de análisis, y que por tanto acaban desembocando en magnitudes dispares del fenómeno, como han mostrado Cantó, Grandin y Del Río (2008) para un conjunto de países europeos. Por un lado, el components approach utiliza la media de las rentas de un periodo para establecer lo que sería la «renta permanente» del hogar, e identifica como pobres crónicos a todos aquellos individuos que viven en hogares que no superan la correspondiente línea de pobreza de esa «renta permanente».

Por otro lado, el spell approach mide la pobreza crónica observando los episodios de pobreza experimentados por los hogares periodo a periodo $y$, especialmente, en la duración total de esas situaciones. La pobreza crónica se establece a través de un segundo umbral de duración de la pobreza, el de la suma de los hogares que permanecen por debajo del umbral de la pobreza un determinado número de periodos respecto al total de periodos en que son observados. Pobre crónico es aquél que pasa, al menos, un porcentaje de tiempo $\tau$ por debajo de la línea de pobreza $z$. Es obvio que la elección de $\tau$ determina el grado de cronicidad o persistencia y condiciona de forma determinante los resultados. El máximo de cronicidad consistiría en tomar como periodo de referencia el número total de periodos observados para los hogares, siendo $\tau=1$ (un individuo sería pobre crónico sólo si pasa el 100\% del tiempo observado en la pobreza), que suele denominarse "pobreza crónica pura». Lo más habitual es definir una cronicidad en la que $\tau$ toma valores de entre 0 y 1 , con lo que se identifica como pobres crónicos a individuos con un menor grado de persistencia en la pobreza que en el supuesto de $\tau=$ 1. Esta es la opción que ha elegido la UE-EUROSTAT en los llamados Indicadores de Laeken, que definen la "pobreza persistente» como el porcentaje de individuos pobres en un año determinado y en dos de los tres anteriores, lo cual equivale a una $\tau=0,75$.

De un modo similar, la OCDE $(2001,2009 a)$ viene proporcionando indicadores nacionales para cuatro niveles de duración de la pobreza según su grado de persistencia a lo largo de un periodo (gráfico 3): 
- Nunca son pobres a lo largo de un periodo (no pobreza).

- Son pobres sólo en uno de los años del periodo considerado (pobreza temporal).

- Son pobres dos de los tres años del periodo (pobreza recurrente).

- Son pobres los tres años del periodo (pobreza persistente).

Con esas definiciones, los datos del gráfico 3 muestran un par de rasgos de la duración de la pobreza: por un lado, que parece existir una asociación positiva entre el nivel promedio de pobreza en los tres años y las tasas de pobreza de mayor duración, las de pobreza persistente y recurrente; por otro lado, que las diferencias relativas entre países en la frecuencia de los niveles de pobreza persistente y recurrente son mucho mayores que las tasas promedio, con lo que se apunta que los patrones de persistencia en la pobreza son mucho más desiguales entre países.

Gráfico 3. Tasas de pobreza temporal, recurrente y persistente, h. 2000 (Pobreza basada en el $50 \%$ de la media, porcentajes para un periodo de tres años)

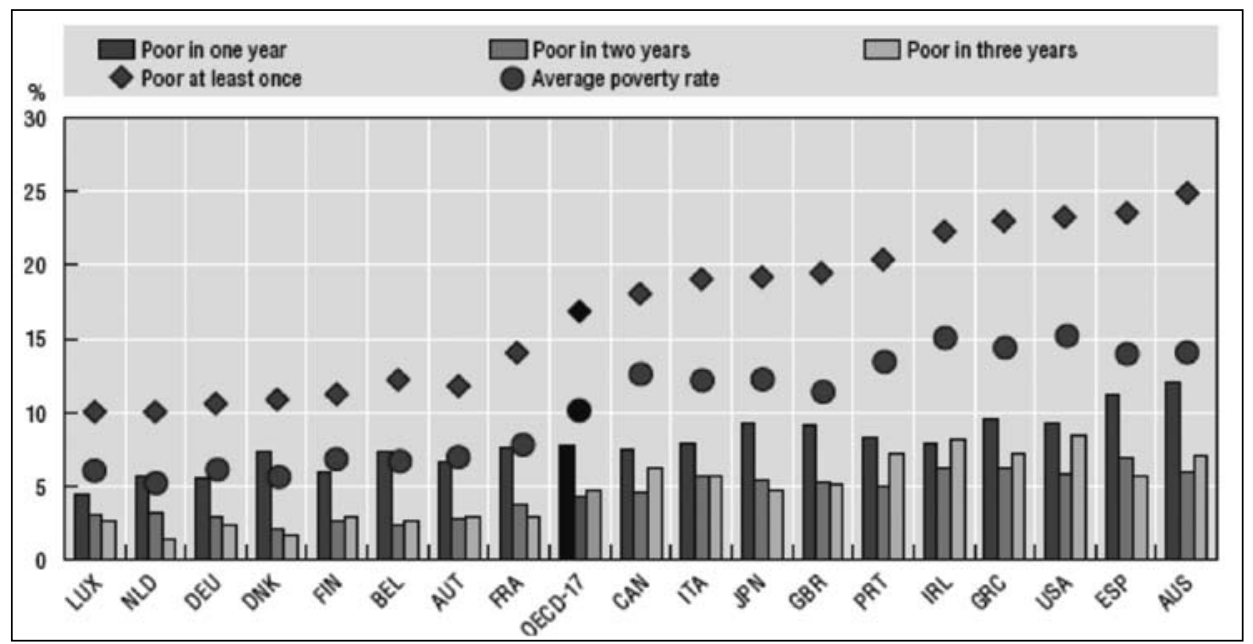

Fuente: tomado de OCDE, 2009a, cap. 5.

Como ya se había anticipado, la elección de enfoque y de grado de persistencia altera sensiblemente los resultados, pero también ayuda a comprender mejor el perfil comparado entre países de la composición de la pobreza. En el cuadro 2 se recogen los resultados para seis países (España, Alemania, Dinamarca, Francia, Portugal y Reino Unido) y un periodo de siete años (1994-2001) muy próximo al del gráfico 3, usando ambos enfoques, el de spells y el de components, e identificando los tres grados de persistencia de la pobreza: temporal, persistente y recurrente. Estos datos muestran, en 
primer lugar, que tanto la composición de la pobreza (tanto por ciento en cada tipo del total de quienes son pobres al menos una vez durante el periodo, recogido en la primera fila para cada país) como la frecuencia de cada tipo de pobreza entre el conjunto de la población (segunda fila), varían de manera muy apreciable según el método y el indicador utilizado. En general, tanto el peso como la frecuencia de la pobreza persistente son mucho más altos cuando se miden con el enfoque de components, hasta el punto de que ambos indicadores se multiplican por cuatro o cinco cuando se usa este segundo método; también con este método, el peso de la pobreza persistente alcanza en los seis países un mínimo de una cuarta parte del total de quienes han sido pobres alguna vez en ese espacio de siete años.

Tabla 2. Tasas de pobreza persistente, recurrente y temporal en algunos países de la UE, 1994-2001

\begin{tabular}{|c|c|c|c|c|c|c|c|c|}
\hline \multirow{3}{*}{ Países } & \multicolumn{4}{|c|}{$\begin{array}{c}\text { Spells approach } \\
(\tau=1)\end{array}$} & \multicolumn{4}{|c|}{$\begin{array}{c}\text { Components approach } \\
\text { (7 años) }\end{array}$} \\
\hline & \multirow[b]{2}{*}{ Persistente } & \multicolumn{2}{|c|}{ Transitoria } & \multirow[b]{2}{*}{ Total } & \multirow[b]{2}{*}{ Persistente } & \multicolumn{2}{|c|}{ Transitoria } & \multirow[b]{2}{*}{ Total } \\
\hline & & $\begin{array}{c}\text { Recurrente } \\
\text { (dos o más } \\
\text { spells) }\end{array}$ & $\begin{array}{l}\text { Temporal } \\
\text { (un spell) }\end{array}$ & & & $\begin{array}{c}\text { Recurrente } \\
\text { (dos o más } \\
\text { spells) }\end{array}$ & $\begin{array}{l}\text { Temporal } \\
\text { (un spell) }\end{array}$ & \\
\hline \multirow{2}{*}{ España } & 6,1 & 41,5 & 52,4 & 100 & 31,5 & 23,8 & 44,7 & 100 \\
\hline & 2,7 & 18,2 & 23,0 & 43,9 & 13,8 & 10,5 & 19,6 & 43,9 \\
\hline \multirow{2}{*}{ Alemania } & 6,8 & 26,8 & 66,4 & 100 & 26,6 & 13,6 & 59,7 & 100 \\
\hline & 1,9 & 7,5 & 18,6 & 18,6 & 7,4 & 3,8 & 16,7 & 28,0 \\
\hline \multirow{2}{*}{ Dinamarca } & 3,5 & 23,0 & 73,5 & 100 & 26,0 & 12,5 & 61,4 & 100 \\
\hline & 1,0 & 6,6 & 21,1 & 28,7 & 7,5 & 3,6 & 17,6 & 28,7 \\
\hline \multirow{2}{*}{ Francia } & 8,4 & 31,4 & 60,1 & 100 & 35,9 & 14,3 & 49,7 & 100 \\
\hline & 2,8 & 10,2 & 19,5 & 32,5 & 11,6 & 4,6 & 16,1 & 32,5 \\
\hline \multirow{2}{*}{ Portugal } & 12,7 & 31,5 & 55.8 & 100 & 41,1 & 15,5 & 43,4 & 100 \\
\hline & 5,6 & 13,8 & 24,6 & 44,0 & 18,1 & 6,8 & 19,1 & 44,0 \\
\hline Reino & 8,0 & 29,1 & 62,9 & 100 & 40,4 & 10,9 & 48,6 & 100 \\
\hline Unido & 2,8 & 10,5 & 22,7 & 36,6 & 14,6 & 3,9 & 17,6 & 36,1 \\
\hline
\end{tabular}

Fuente: EHP (tomado de Cantó, Grandín y Del Río, 2008).

Los datos de la tabla 2 permiten también confirmar que el patrón de cronicidad de los países no se corresponde a niveles de pobreza estática. Portugal y España poseen altas y similares tasas promedio de pobreza (gráfico 3), pero Portugal alcanza un nivel de persistencia de la pobreza muy superior, el 12,7\% con el primer indicador, y el $41,1 \%$ con el segundo. El Reino Unido presenta el patrón inverso, con una tasa promedio más baja que la de España; sin embargo, posee una frecuencia y un peso de la pobreza persistente superior a la de España, con ambos indicadores. En situación diferente está Dinamarca, que posee una tasa promedio muy baja, acompañada también por un patrón de pobreza de carácter mucho más transitorio, ya que presenta, con cualquiera de 
los indicadores, la proporción más baja de pobreza persistente y la más alta de pobreza temporal (en este caso, sólo un año de los siete).

\subsection{Factores que explican la dinámica de la pobreza}

La gama de factores que pueden determinar la dinámica de la pobreza no son diferentes de los que explican su frecuencia con perspectiva transversal; también coinciden con los de la pobreza laboral. Los estudios analizan una gama de tres tipos básicos de factores explicativos, tomados dinámicamente, es decir, como cambios o eventos:

a) Los demográficos que varían la composición del hogar, debidos bien al nacimiento de un hijo, bien a la formación de nueva pareja, al fallecimiento, a la ruptura de la pareja, a la monoparentalidad o a la viudedad.

b) Los relativos a la participación laboral de los hogares.

c) Los que afectan a las rentas del hogar, sean salarios, transferencias sociales $u$ otras fuentes de renta.

Los estudios que han analizado la influencia de estos factores suelen coincidir en la existencia de un patrón comparado bastante estable: los cambios en la estructura de los hogares, y muy en particular la monoparentalidad, constituyen la principal explicación de la persistencia en la pobreza en EE.UU., mientras que los cambios en la participación laboral y en las transferencias de renta dominan en los países europeos (OCDE, 2001; Whelan, Layte y Maitre, 2003; Fourage y Layte, 2005; Ayala y Sastre 2007).

Sin embargo, la explicación de la movilidad presenta un par de hechos que no encuentran suficiente explicación, como se puede vislumbrar en los datos del gráfico 4. Por un lado, existe un alto peso de otros factores o eventos no identificados (no se corresponden a ninguno de los tres habituales) asociados a la entrada en situaciones de pobreza, que alcanza el 30\% para el conjunto de la OCDE y hasta cerca del $50 \%$ en otros países, como EE.UU. Por otro, el patrón de eventos determinantes no parece asociarse a las variantes de regímenes de bienestar, mucho más allá de estas diferencias entre EE.UU. y Europa; por ejemplo, el Reino Unido experimenta una influencia de los eventos de la estructura familiar que se parece más a la de Finlandia o Dinamarca que a países de la variante anglosajona; e Italia y España apenas se diferencian en este aspecto de la mayoría de los países de la variante continental, a no ser por un efecto algo menor de las transferencias públicas. 
Gráfico 4. Tipos de cambios que ocasionan entradas en situación de pobreza en países de la OCDE, h. 2000 (Porcentaje de personas con un determinado evento cuando entran en pobreza)

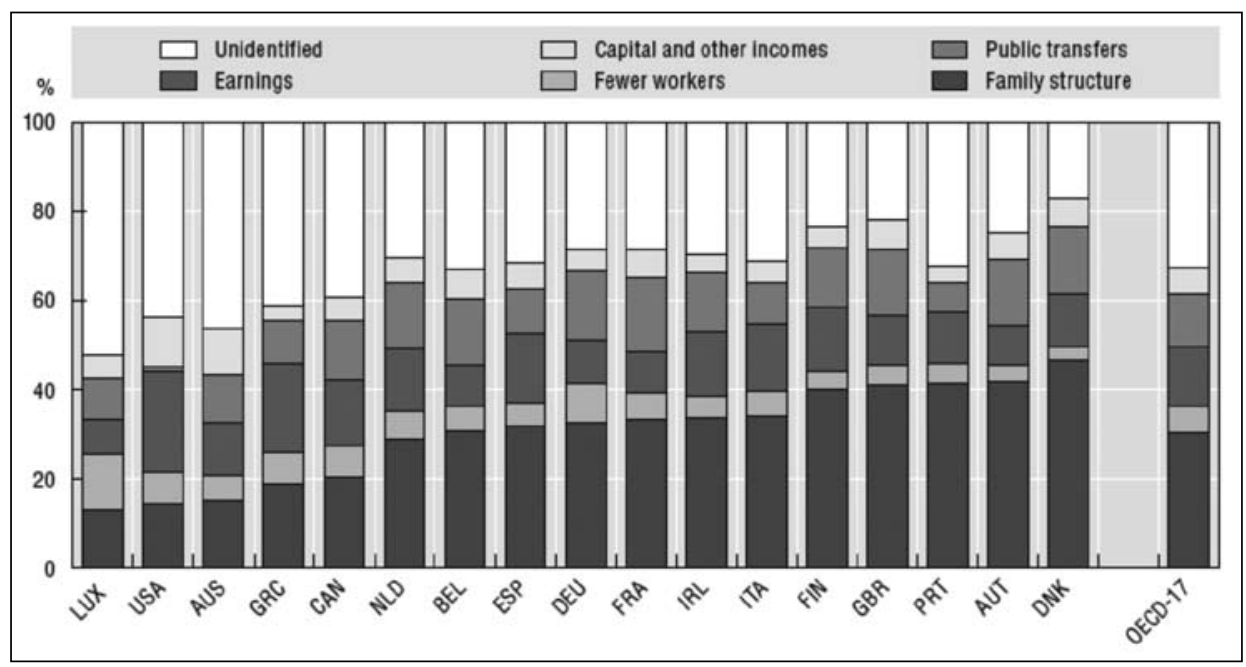

Fuente: Tomado de OCDE, 2009a, cap. 6.

Los datos del gráfico 4 también dejan claro que en España, como en otros países de la variante continental-mediterránea de régimen de bienestar (Italia y Grecia), es más frecuente que las variaciones en los salarios (no causadas por cambios en el número de miembros del hogar que trabajan) se relacionen con entradas en la pobreza, asemejándose en esto más a EE.UU. y a Canadá que a los países europeos. Esta mayor influencia de los cambios en las rentas salariales en el caso español está bien confirmado por otros estudios (Cantó, Grandin y Del Río, 2008). Los eventos demográficos suponen sólo alrededor de un $16 \%$ de las razones para salir de la pobreza y un 13\% de las que suceden cuando se entra en ella, en los hogares cuyo sustentador principal está en edad potencialmente activa, mientras que el resto de las causas están relacionadas con cambios laborales de los miembros del hogar o con transferencias recibidas por prestaciones públicas; y del total de transiciones relacionadas con estos dos tipos de eventos, los cambios laborales se asocian al $75 \%$ de las transiciones de entrada y al $69 \%$ en las de salida, mientras que las transiciones vinculadas a cambios en las transferencias públicas sólo afectan al $4 \%$ tanto de entradas como de salidas en la pobreza. 


\section{Datos y metodologíaः el análisis longitudinal en la UE- SILC}

La disponibilidad de una fuente estadística armonizada a nivel europeo como la UESILC permite aproximar ambas dimensiones, la de ocupación y la de rentas, para las mismas poblaciones. No obstante, también se plantean limitaciones en el uso de esta fuente por varios motivos: la discontinuidad de las series con la antigua European Community Household Panel (ECHP)-Panel de Hogares de la Unión Europea (PHOGUE), el carácter reciente de esa fuente, que ofrece los datos con diferente calendario para algunos países, y, en general, los problemas de calidad de la información sobre rentas en las fuentes basadas en encuestas.

Por otra parte, la categoría de trabajador pobre admite diferentes definiciones operativas. En este trabajo se adopta la aplicada por EUROSTAT, según la cual son trabajadores pobres las personas «principalmente ocupadas» en el año de referencia que pertenezcan a hogares cuya renta disponible equivalente (después de transferencias sociales) esté por debajo del umbral del $60 \%$ de la renta media del país. El componente de la renta equivalente, en esta definición, que permite ajustar el umbral de pobreza por el tamaño y la composición del hogar, se calcula aplicando la escala de la $\mathrm{OCDE}^{2}$. El otro componente, la condición de «principalmente ocupado», es obtenido a partir del concepto de most frequent activity status (MFAS), que es aquél definido como «la situación de los individuos que declaran haber estado ocupados más de la mitad del número total de meses del año al que se refiere el calendario de actividades de la encuesta.» Ese calendario de actividades para todos los miembros adultos del hogar, que se incluía ya en el ECHP, se refiere al año completo anterior al de la encuesta, para hacer coincidir la información de MFAS con la de las rentas del hogar. Si el calendario de actividades informa de un individuo que declara haber trabajado seis meses y haber estado en paro el tiempo restante del año, resultará clasificado como empleado y, consiguientemente, si declara un periodo de desempleo de siete meses, se clasificaría como parado, y por lo tanto escaparía a las estadísticas referidas a trabajador pobre. Esta definición europea se centra, por tanto, en individuos que han trabajado efectivamente por encima de ese umbral, bien por cuenta propia o por cuenta ajena, y se diferencia de otros indicadores internacionales, como el que usa la OCDE (2009b), en el que la condición de trabajador pobre se refiere a todos los individuos en un hogar pobre (con diferente escala y umbral de pobreza) en el que al menos un adulto ha estado ocupado alguna vez durante el año.

2 Esta escala otorga un peso de 1 al primer adulto del hogar, de 0,5 a los demás miembros del hogar de 14 años de edad o más, y de 0,3 a los menores de esa edad. 
Por lo que se refiere al análisis dinámico, la UE-SILC, que comienza en 2004, tiene la ventaja de su total comparabilidad en el marco europeo, al ser una de las operaciones estadísticas acordadas y armonizadas por los países de la UE (extendida también a otros) relativas a la distribución de ingresos y la exclusión social en el ámbito europeo (INE, 2004). Sin embargo, frente a la investigación de panel de la que es heredera, la UE-SILC presenta ciertas limitaciones para el análisis longitudinal. El esquema muestra de la UE-SILC es de cuatro paneles anuales, de tal forma que las personas de cada uno de ellos permanecen en la muestra durante cuatro años consecutivos ${ }^{3}$. Por ello, el seguimiento a lo largo de cuatro años del grupo de rotación supone trabajar con un cuarto de la muestra original. Cuando se analiza un fenómeno con una incidencia minoritaria como el de pobreza entre los trabajadores, más aún si se limita a los asalariados, la muestra puede perder representatividad. El problema se agrava en los países con muestras más reducidas y en aquellos con una incidencia de trabajadores pobres más escasa.

El análisis dinámico de la pobreza necesita al menos tres años consecutivos para conseguir un periodo que permita una distinción entre los diferentes niveles de duración, lo que condiciona también la selección de países, para los que hay ya disponible una serie de tres años de la UE-SILC con muestra suficiente. Además, en esta fuente las variables de renta y la intensidad laboral del hogar, que necesitan informar de años completos, se refieren al año anterior, mientras que otras variables se refieren al año de la encuesta. Por todo ello, en este análisis del periodo 2004-2006, la movilidad respecto a situaciones de pobreza entre 2004 y 2005 se ha realizado con las oleadas de 2005 y 2006 respectivamente, mientras que para las variables de estructura del hogar se ha acudido a 2004 y 2005.

Una dificultad añadida en el análisis dinámico de la pobreza es el empleo de las ponderaciones, ya que cada oleada tiene un coeficiente de ponderación distinto. Eso hace que haya que elegir diferentes años de ponderación; en las transiciones descriptivas (árboles de movilidad) se ha optado por ponderarlo en el último año observado, el 2006; en el análisis descriptivo de los factores de influencia en cinco países de la UE se ha utilizado el inicio del cambio, el año 2005; el mismo año que se ha empleado en el análisis logístico. Para asegurar que el empleo de uno u otro factor de ponderación no distorsiona el análisis, se cotejaron ambos resultados, siendo las diferencias de porcentajes en ningún caso superiores a un punto, y en general en torno al medio punto.

Para analizar los factores influyentes en las transiciones de pobreza se han elaborado algunas variables dependientes, referidas a factores demográficos, de participación laboral o de rentas, y definidas del siguiente modo sobre la base de los ficheros longitudinales de la UE-SILC.

3 Las 2.000 secciones censales que suponen la primera unidad muestra en España se han dividido en cuatro grupos, a los que se les ha denominado «grupos de rotación», que son los cuatro paneles que componen la muestra. 
Menores dependientes. Puesto que en esos ficheros no se dispone de la variable de tipo de hogar (HX060), se toma la del tamaño del hogar equivalente (HX050), que permite acceder al número de menores en el hogar. Además, la variable HX050 está referida al final del periodo de referencia de los ingresos. El cambio del número de dependientes es la diferencia entre un año y otro.

Intensidad laboral del hogar. Esta variable tampoco se proporciona directamente en los ficheros. Es el resultado de usar las relativas al año de nacimiento (RB080), la actividad (RB210) y la convivencia con alguno de los progenitores (RB220_F y RB230_F) para el denominador, el número de personas potencialmente activas en el hogar. El numerador se halla a partir del conjunto de meses ocupados de dichos sujetos (PL210A-L). El cambio en la intensidad laboral es la diferencia entre un año y otro.

Ingresos salariales. Se elabora un índice de ingresos salariales como el porcentaje de los ingresos salariales (PY010+PY020) respecto al umbral de pobreza del país y el año, de forma que si el índice es 100 se ha ganado lo mismo que el umbral de pobreza, si es 200 se ha ganado el doble, y si es 50 se ha ganado la mitad.

\section{Persistencia en la pobreza laboral}

En este apartado se presentan un conjunto de resultados relativos a la composición de la pobreza laboral por su nivel de duración o persistencia. Estos resultados se refieren al periodo de tres años para el que se dispone de datos, de un conjunto de países de la UE (2004-2006); no se incluyen Alemania ni el Reino Unido, ya que en ambos casos sólo se dispone de dos años.

En primer lugar, se presenta la frecuencia de los diferentes niveles de persistencia en situación de pobreza laboral: permanente, recurrente y temporal (tabla 4). Estos datos muestran unos perfiles en la composición de la pobreza laboral que coinciden sólo parcialmente con los que se han mostrado para la pobreza laboral estática y para la dinámica de la pobreza total. En primer lugar, el indicador que recoge la proporción de de ocupados que han experimentado la pobreza alguna vez a lo largo de los tres años, cualquiera que fuera su duración, eleva considerablemente, hasta casi duplicar, la proporción de quienes sufren esa situación según los tasas estáticas año a año en unos países (los mediterráneos principalmente), pero no en otros, como es el caso de los nórdicos. Por otra parte, refleja similares patrones comparados a los de los indicadores estáticos, tanto de pobreza como de pobreza laboral: los países de la variante mediterránea tienen los niveles altos y similares - con España como en el nivel más alto de todos los países-, mientras que los de la variante escandinava poseen los niveles más bajos, y los de la variante continental, los niveles medios. 
Tabla 4: Tasas de pobreza según su duración en países de la UE, 2004-2006

\begin{tabular}{|l|c|c|c|c|c|}
\hline & $\begin{array}{c}\text { Al menos } \\
\text { un año en la } \\
\text { pobreza }\end{array}$ & Permanente & Recurrente & Temporal & No pobreza \\
\hline España & 17,78 & 2,92 & 4,27 & 10,59 & 82,22 \\
\hline Portugal & 15,57 & 6,39 & 4,16 & 5,02 & 84,43 \\
\hline Grecia & 18,8 & 5,53 & 4,93 & 8,34 & 81,20 \\
\hline Italia & 14,69 & 3,44 & 4,12 & 7,14 & 85,31 \\
\hline Suecia & 6,79 & 1,53 & 1,14 & 4,12 & 93,21 \\
\hline Noruega & 7,96 & 1,48 & 1,95 & 4,53 & 92,04 \\
\hline Finlandia & 6,45 & 1,07 & 1,14 & 4,24 & 93,55 \\
\hline Dinamrca & 6,51 & 0,51 & 1,18 & 4,82 & 93,49 \\
\hline Luxemburgo & 11,22 & 2,94 & 4,30 & 3,98 & 88,78 \\
\hline Francia & 11,22 & 1,19 & 2,85 & 7,18 & 88,78 \\
\hline Austria & 13,85 & 0,85 & 3,03 & 9,97 & 86,15 \\
\hline Bélgica & 6,7 & 0,25 & 1,30 & 5,15 & 93,30 \\
\hline Irlanda & 10,07 & 1,10 & 2,54 & 6,43 & 89,93 \\
\hline Estonia & 13,74 & 2,18 & 2,63 & 8,93 & 86,26 \\
\hline
\end{tabular}

Sin embargo, la composición por niveles de duración o persistencia ya no parece responder a ese patrón de relación con las variantes de bienestar. En el grupo de países mediterráneos, España posee una composición de la pobreza laboral mucho más temporal que la de Italia, Grecia y Portugal: en España, la suma del porcentaje de quienes experimentan situaciones de pobreza persistente y recurrente alcanza poco más de una tercera parte del total, mientras que en el resto de países suma entre la mitad y las dos terceras partes. Otra diferencia es que España casi triplica el porcentaje de Suecia en quienes han padecido al menos un año de pobreza, luego apenas llega a duplicar la tasa de pobreza persistente de Suecia. Los países continentales tienen una posición intermedia entre los países nórdicos y los mediterráneos, con tasas de permanencia bastante bajas, y recurrencia y temporalidad intermedias.

Tabla 5. Tasas de entrada y salida en la pobreza de ocupados 2004-2006

\begin{tabular}{|l|c|c|c|c|}
\hline & $\mathbf{2 0 0 4}$ & \multicolumn{2}{|c|}{2005} & 2006 \\
\hline & Tasa salida & Tasa entrada & Tasa salida & Tasa entrada \\
\hline Austria & 72,37 & 4,81 & 61,29 & 4,00 \\
\hline Bélgica & 70,03 & 2,21 & 77,30 & 2,35 \\
\hline Dinamarca & 42,03 & 2,76 & 86,36 & 3,63 \\
\hline Estonia & 61,21 & 2,66 & 47,15 & 2,78 \\
\hline España & 51,69 & 4,82 & 52,57 & 4,96 \\
\hline Finlandia & 62,72 & 1,50 & 44,19 & 1,28 \\
\hline Francia & 60,73 & 3,41 & 57,83 & 3,19 \\
\hline Grecia & 37,48 & 4,28 & 33,51 & 3,87 \\
\hline
\end{tabular}




\begin{tabular}{|l|l|l|l|l|}
\hline Irlanda & 66,67 & 1,94 & 54,12 & 2,25 \\
\hline Italia & 38,22 & 3,81 & 42,60 & 3,76 \\
\hline Luxemburgo & 30,75 & 3,41 & 30,45 & 2,32 \\
\hline Noruega & 57,09 & 2,35 & 34,18 & 1,41 \\
\hline Portugal & 23,19 & 2,50 & 29,01 & 3,24 \\
\hline Suecia & 49,96 & 0,82 & 26,82 & 0,71 \\
\hline Alemania & & & 67,52 & 3,18 \\
\hline Reino Unido & & & 62,36 & 4,73 \\
\hline
\end{tabular}

Fuente: Elaboración propia a partir de la Encuesta de Condiciones de Vida 2004-2006

La información sobre flujos de entrada y salida en pobreza laboral (tabla 5) confirma ese patrón de una pobreza laboral con niveles altos de movilidad para el caso español, algo menor desde luego que el de los países nórdicos y continental, pero muy superior al resto de los países mediterráneos. Los árboles de movilidad complementan esa imagen (figura 1) y permiten destacar que, a pesar de ese alto nivel de pobreza temporal, la pobreza persistente y recurrente de ocupados no es despreciable. En ese corto plazo de tres años, quienes salen de la pobreza en 2005 tienen pocas probabilidades de volver a ella en 2006, ya que sólo uno de cada cinco lo hacen; pero quienes permanecen el segundo año en situación de pobreza tienen muchas más probabilidades de permanecer el tercer año, ya que tres de cada cinco de los que seguían siendo pobres en 2005 continúan siéndolo en 2006.

Figura 1. Transiciones desde la situación de trabajadores pobre en España 20042006

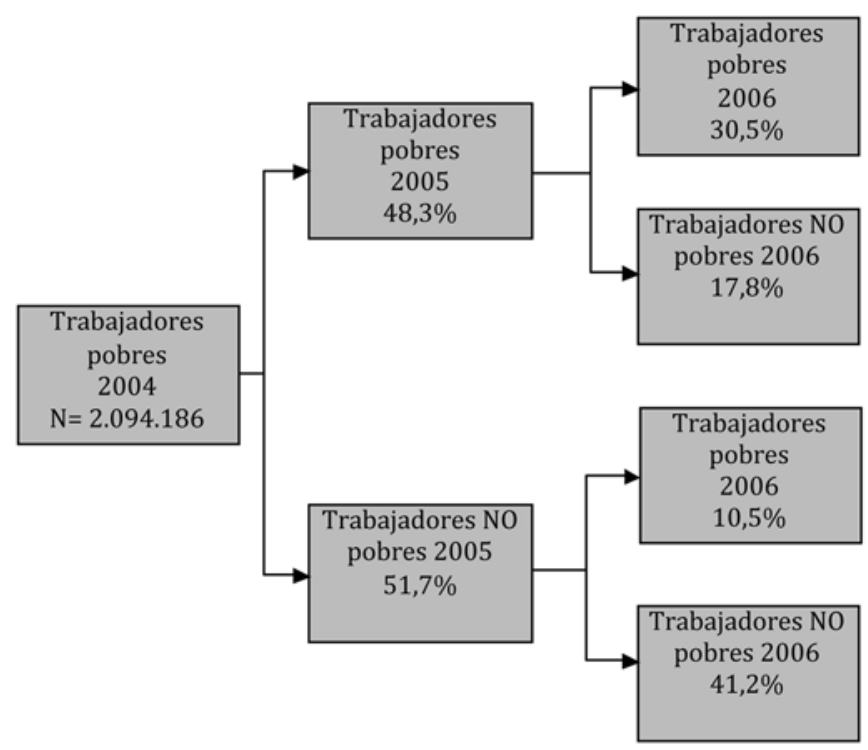

Fuente: elaboración propia a partir de la Encuesta de Condiciones de Vida 2004-2006. 


\section{Análisis de la movilidad de la pobreza laboral en España}

En este apartado se van a analizar, por medio de modelos de regresión logística, cuáles son los principales determinantes de las transiciones de salida y entrada en la pobreza laboral para el caso de España. Para las transiciones de salida, se ha tomado como población de referencia la constituida por los trabajadores que eran pobres en 2004, y la variable dependiente de los modelos asume los valores de «1» si ha dejado de ser pobre en 2005, y «0» si no ha sido así. En las transiciones de entrada en la pobreza, el colectivo de estudio son todos los trabajadores que no son pobres en 2004, y se analiza la probabilidad de entrar en la pobreza en 2005; por tanto, los valores de la variable dependiente son «1», si entra en la pobreza, $y$ «0», si no entra en tal situación. Así mismo, los modelos se han elaborado en primer lugar para los trabajadores en general, tanto por cuenta propia como por cuenta ajena, y en segundo lugar para los asalariados.

Las variables independientes que se incluyen en estos modelos de regresión son relativas a los tres tipos de factores potencialmente determinantes. En primer lugar, dos variables de estructura de los hogares, una sobre la presencia o no de menores dependientes en el hogar y otra sobre cambios en el número de menores dependientes durante el periodo. En segundo lugar, las variables sobre participación laboral, que incluyen variables sobre características del empleo de los ocupados (categoría profesional, jornada semanal, la condición de asalariado o no asalariado para el modelo de ocupados, y la de contrato fijo o temporal para el modelo de asalariados) y sobre si ha habido cambios en la intensidad de la participación laboral del conjunto de activos del hogar durante el periodo. En tercer lugar, una variable relativa a las rentas, definida como cambios en ingresos por trabajo de 2005 respecto a 2004. Además, se han incluido una serie de variables de control de características sociodemográficas de los individuos, tales como la edad, el sexo y el nivel de estudios.

\subsection{Transiciones de salida de la pobreza}

Los resultados del modelo para estas transiciones indican una influencia de todas las variables incorporadas sobre las probabilidades de salir de la pobreza, aunque sea muy variable en dirección e intensidad (tabla 6). El aumento en el número de menores dependientes y la intensidad laboral de los hogares son las variables de mayor influencia; la primera en el sentido de que dificulta la salida de la pobreza, la segunda en la dirección de favorecerla. La influencia de esta segunda variable es singularmente intensa, más que los cambios en la propia intensidad de esa participación. Esos efectos, además, son aún más notables entre los asalariados que entre el conjunto de ocupados. 
Esos resultados, que dan una influencia tan sólida de la presencia de menores dependientes y de la intensidad laboral de los hogares, contrastan con la casi nula influencia que se observa de la variable de variación en los ingresos laborales. El incremento de los mismos apenas mejora la probabilidad de salir de la pobreza; la facilita muy ligeramente sólo para los asalariados, y no para el conjunto de los ocupados. Puede que este resultado tenga que ver con que, en transiciones entre sólo dos años, los incrementos de rentas laborales sean de escasa magnitud y tengan muy poca influencia, al igual que sucede en las restantes variables, dada especialmente una determinada intensidad laboral.

Las variables sociodemográficas muestran influencias que pueden considerarse esperables. La probabilidad de salir de la pobreza laboral se ve afectada negativamente entre los varones, los más jóvenes y los que no tienen educación universitaria, respecto a los que sí la tienen.

\section{Tabla 6. Regresión logística de las transiciones de salida de la pobreza labo- ral en España 2004-2005}

\begin{tabular}{|c|c|c|}
\hline & Ocupados & Asalariados \\
\hline Varón (Ref. mujer) &,$- 114^{* * *}$ & $-024^{* * *}$ \\
\hline Grupos de edad (Ref. Más de 50) & $* * *$ & $* * *$ \\
\hline De 16 a 30 &,$- 447^{* * *}$ &,$- 257^{* * *}$ \\
\hline De 31 a 40 & $-304^{* * *}$ & $-278^{\star * *}$ \\
\hline De 41 a 50 &, $058^{* * *}$ &,$- 288^{* * *}$ \\
\hline Nivel educativo (Ref. Universitario) & $* * *$ & $* * *$ \\
\hline Primarios &,$- 993^{* * *}$ & $-1,523^{* * *}$ \\
\hline Secundarios $1 .^{\mathrm{a}}$ etapa &,$- 599 * * *$ &,$- 996^{* * *}$ \\
\hline Secundarios $2 .^{\text {a }}$ etapa &,$- 080^{* * *}$ &,$- 010^{* * *}$ \\
\hline Existencia de niños dependientes (Ref. Sí) &, $292^{* * *}$ &, $374^{* * *}$ \\
\hline Intensidad laboral & $3,353^{* * *}$ & $3,862^{* * *}$ \\
\hline $\begin{array}{l}\text { Cambio en el número de dependientes (Ref. Sin } \\
\text { cambio) }\end{array}$ & $* * *$ & $* * *$ \\
\hline Más dependientes & $-1,345^{* * *}$ & $-2,232^{* * *}$ \\
\hline Menos dependientes &, $927^{* * *}$ & $1,533^{* * *}$ \\
\hline Cambio en la intensidad laboral (Ref. Disminuye) & $* * *$ & $* * *$ \\
\hline No cambia &,$- 602^{* * *}$ & $-1,015^{* * *}$ \\
\hline Aumenta &, $156^{* * *}$ &, $249^{* * *}$ \\
\hline $\begin{array}{l}\text { Categoría ocupacional (Ref. Trabajadores no } \\
\text { cualificados) }\end{array}$ & $* * *$ & $* * *$ \\
\hline - Ejecutivos, empresarios y técnicos & $900^{* * *}$ & $3,223^{* * *}$ \\
\hline - Administrativos y técnicos de apoyo &,$- 737^{* * *}$ & $-2,189^{* * *}$ \\
\hline - Trabajadores de los servicios &,$- 357^{* * *}$ & $-203^{* * *}$ \\
\hline - Trabajadores cualificados de la agricultura &, $529^{* * *}$ &,$- 417^{* * *}$ \\
\hline - Trabajadores cualificados de la industria & $485^{* * *}$ &, $206^{* * *}$ \\
\hline
\end{tabular}




\begin{tabular}{|l|c|c|}
\hline- Operadores, montadores &, $008^{* * *}$ &, $334^{* * *}$ \\
\hline Situación profesional (Ref. asalariado) & $-733^{* * *}$ &, $087^{* * *}$ \\
\hline Tipo de contrato (Ref. Contrato temporal) & &, $026^{* * *}$ \\
\hline Horas trabajadas a la semana (Ref. Más de 30 horas) &,$- 195^{* * *}$ &, $013^{* * *}$ \\
\hline Incremento en los ingresos & & $-1,450^{* * *}$ \\
\hline Constante & $-2,064^{* * *}$ & \\
\hline TOTAL N $=\quad-2$ Log Likelihood & & \\
\hline
\end{tabular}

\subsection{Transiciones de entrada en la pobreza}

Los modelos que analizan la entrada en la pobreza proporcionan resultados que van sólo parcialmente en la dirección esperada (tabla 7). La probabilidad de transitar hacia una situación de pobreza laboral se ve también fuertemente influida por la presencia de menores dependientes y por la participación laboral de los hogares, pero en este caso la influencia de esta segunda variable es mucho más nítida que en las transiciones de salida. La mayor intensidad en la participación laboral de los adultos es, con diferencia, la variable más influyente, pues reduce el riesgo de entrada en la pobreza; en esta transición, el aumento en esa participación produce un mayor efecto a la hora de evitar el riesgo de entrar, de lo que lo tenía en favorecer la posibilidad de salir. En estas transiciones, el efecto de los cambios en los ingresos laborales alcanza aún una influencia menor que en las transiciones de salida. Sin embargo, sí se muestra más influyente la variable de jornada laboral, que claramente empuja hacia entradas en la pobreza cuando se trabaja en jornada no completa (menos de 30 horas).

Sin embargo, la variable sobre los cambios en el número de menores dependientes muestra efectos contrarios a los esperados: la presencia de más niños no aumenta el riesgo de entrar en la pobreza, e incluso lo reduce ligeramente respecto a los hogares sin cambios. Este efecto, por paradójico que pueda parecer, es verosímil; significa que para quien ya está en una situación de pobreza, la llegada de un nuevo menor al hogar perjudica mucho sus probabilidades de salir de la pobreza, mientras que para quien no está en situación de pobreza, no le perjudica. La conjetura más probable es que los hogares en situación de pobreza muy probablemente acumulen ya rasgos de participación laboral muy escasa; algo más improbable sería, pues, que la situación empeorara con la llegada de un nuevo menor. En cambio, quienes no se encuentran en situación de pobreza, probablemente ya disfrutan de mejores intensidades laborales, que no se ven perjudicadas por la llegada de un nuevo dependiente.

Las variables referidas a características de los empleos o a rasgos sociodemográficos de los individuos proporcionan resultados de influencias bastante similares a los de las transiciones de salida. Cabe destacar, quizá, que ahora los trabajadores manuales 
sin excepción se asocian a entradas en la situación de pobreza, lo mismo que aquellos asalariados con contratos temporales en sus empleos.

Tabla 7. Regresión logística de las transiciones de entrada en la pobreza laboral en España 2004-2005

\begin{tabular}{|c|c|c|}
\hline & Ocupados & Asalariados \\
\hline Varón (Ref. mujer) &, $094^{* * *}$ &,$- 302^{* * *}$ \\
\hline Grupos de edad (Ref. Más de 50) & $* * *$ & $* * *$ \\
\hline De 16 a 30 &, $149^{* * *}$ & $391^{* * *}$ \\
\hline De 31 a 40 & $436^{* * *}$ & ,639*** \\
\hline De 41 a 50 &, $212^{* * *}$ &, $069^{* * *}$ \\
\hline Nivel educativo (Ref. Universitario) & $* * *$ & $* * *$ \\
\hline Primarios & $641^{\star \star *}$ & $687^{* * *}$ \\
\hline Secundarios $1 .^{\mathrm{a}}$ etapa &,$- 034^{* * *}$ &, $030^{* * *}$ \\
\hline Secundarios $2 .^{a}$ etapa &,$- 096^{* * *}$ &,$- 475^{* * *}$ \\
\hline Existencia de niños dependientes (Ref. Sí) &,$- 471^{* * *}$ &,$- 349^{* * *}$ \\
\hline Intensidad laboral & $-2,125^{* * *}$ & $-3,446^{* * *}$ \\
\hline $\begin{array}{l}\text { Cambio en el número de dependientes (Ref. Sin } \\
\text { cambio) }\end{array}$ & $* * *$ & $* * *$ \\
\hline Más dependientes &,$- 222^{* * *}$ &,- 212 \\
\hline Menos dependientes &, $920^{* * *}$ & $1,310^{* * *}$ \\
\hline Cambio en la intensidad laboral (Ref. Disminuye) & $* * *$ & \\
\hline No cambia &,$- 074^{\star * *}$ &,$- 126^{\star * *}$ \\
\hline Aumenta &,$- 339^{* * *}$ &,$- 224^{* * *}$ \\
\hline \multicolumn{3}{|l|}{$\begin{array}{l}\text { Categoría ocupacional (Ref. Trabajadores no } \\
\text { cualificados) }\end{array}$} \\
\hline - Ejecutivos, empresarios y técnicos &,$- 859^{* * *}$ & $-2,392^{* * *}$ \\
\hline - Administrativos y técnicos de apoyo &,$- 729^{* * *}$ &,$- 440^{* * *}$ \\
\hline - Trabajadores de los servicios &, $150^{\star \star *}$ &, $187^{* * *}$ \\
\hline - Trabajadores cualificados de la agricultura &,$- 014^{* * *}$ &, $147^{* * *}$ \\
\hline - Trabajadores cualificados de la industria &, $608^{* * *}$ & $947^{* * *}$ \\
\hline - Operadores, montadores &, $368^{* * *}$ &, $790^{\star * *}$ \\
\hline Situación profesional (Ref. asalariado) & $1,138^{* * *}$ & \\
\hline Tipo de contrato (Ref. Contrato temporal) & &,$- 103^{* * *}$ \\
\hline Horas trabajadas a la semana (Ref. Más de 30 horas) & $311^{* * *}$ & $462^{* * *}$ \\
\hline Incremento en los ingresos & &,$- 006^{* * *}$ \\
\hline Constante &, $058^{* * *}$ &,$- 037^{* * *}$ \\
\hline -2 Log Likelihood & & \\
\hline
\end{tabular}




\section{Conclusiones}

El estudio de la dinámica de la pobreza laboral confirma la alta movilidad en este tipo de situaciones. Esa movilidad presenta perfiles que no se corresponden tan claramente con los regímenes de bienestar, tal y como ocurre cuando se analizan indicadores transversales de pobreza. El caso español es significativo en ese sentido, ya que su alto nivel de pobreza laboral se compone de proporciones de pobreza temporal y de tasas anuales de entrada y salida en situaciones de pobreza mucho más altas que las que se observan en otros países de la variante mediterránea.

La información sobre flujos de entrada y salida en pobreza laboral en España permiten también comprobar que, a pesar del alto grado de movilidad, el riesgo de permanecer en esa situación es sensiblemente más alto que el riesgo de retornar a ella tras haber salido. Un $40 \%$ de quienes eran pobres en el primer año observado, continuaban siéndolo en el segundo año, y de ellos, dos quintas partes permanecían en situación de pobreza durante el tercer año.

El análisis de los determinantes de las transiciones de entrada y salida en la pobreza laboral confirma, también para el caso español, que la baja intensidad en la participación laboral de los hogares es el factor que más influye en el riesgo de permanecer en la pobreza o de no salir de ella; por el contrario, los cambios en las rentas laborales no parecen ejercer una influencia apreciable. El aumento en el número de menores dependientes en el hogar influye también de manera intensa en el riesgo de permanecer en la pobreza, pero no parece causar una mayor probabilidad de entrada.

En este trabajo no se ha analizado la influencia de otro de los factores habitualmente determinantes del riesgo de permanecer o bien de la posibilidad de salir de la pobreza: las transferencias públicas de rentas. El bajo porcentaje de hogares que reciben estas rentas en España hace que las submuestras de hogares de ocupados bajo riesgo de pobreza sean escasas para realizar análisis multivariables de la influencia de ese factor. En todo caso, resulta claro que el análisis de cómo afectan las transferencias de rentas a esos hogares en los que se concentra el riesgo de pobreza laboral en España, los hogares de baja e inestable intensidad laboral que tienen niños, es una de las líneas más sugerentes de progreso de la investigación sobre dinámica de pobreza.

\section{Bibliografía}

Allegre, G. (2008), «Working poor in France: an explanatory comparative analysis», comunicación presentada en RECWOWE Task 03.11 Meeting, 14 y 15 de noviembre de 2008. Universidad de Oviedo. 
Andress, H. J.y Lohmann, H. (2008), The Working Poor in Europe. Employment, Poverty and Globalization, Edward Elgar, Cheltenham.

Ayala, L. y Sastre, M. (2008), «The structure of income mobility: Empirical evidence from five UE countries», en Empirical Economics, 35-3, págs. 451-473.

Bárcena, E. y Cowell, F. A. (2006), «Static and Dynamic Poverty in Spain, 19932000», en Hacienda Publica Española / Revista de Economia Publica, 179: 51-78.

Cantó, O; Gradín, C., y Del Río, C. (2006) «Poverty statics and dynamics: Does the accounting period matter?», en International Journal of Social Welfare, 15 (3): 209-218.

Duncan, G.J.; Gustafsson, B.; Hauser, R.; Schmauss, G.; Messinger, H.; Muffels, R.; Nolan, B., y Ray, J. C. (1993), «Poverty dynamics in eight countries», en Journal of Population Economics, 6: 215-234.

Fourage, D. y Layte, R. (2005) «Welfare regimes and poverty dynamics», en Journal of Social Policy, 34: 1-20.

García, I.; Gutiérrez, R., e Ibáñez, M. (2008), «In-work poverty in Spain», comunicación presentada en RECWOWE Task 03.11 Meeting. 14 y 15 de noviembre de 2008. Universidad de Oviedo.

Goerne, A. y Fraser, N. (2008), «In-work poverty in the UK: An analysis of UE-SILC data», comunicación presentada en RECWOWE Task 03.11 Meeting. 14 y 15 de noviembre de 2008. Universidad de Oviedo.

Lohmann, H. (2008), «The working poor in European welfare states: Empirical evidence from a multilevel perspective», en Andress, H.J. y LohmanN, H. (2008), págs. 47-74.

Lohmann, H. y Marx, I. (2008), "The different faces of in-work poverty across welfare state regimes», en Andress, H. J. y Lohmann, H. (2008), págs. 17-46.

OCDE (2001), Employment Outlook 2001, cap. 2, París.

OCDE (2009a), Growing Inequality, París.

OCDE (2009b), Employment Outlouk 200, cap. 3, París.

Peña-Casas, R. y Latta, M. (2004), Working poor in the European Union. European Foundation for the Improvement of Living and Working Conditions, Dublín.

Whelan, C.; Layte, R. y Maitre, B. (2003), «Persistent income poverty and deprivation in the European Union», en Journal of Social Policy, 32: 1-18. 